\title{
High-Frequency Electromagnetic Field Coupling to Small Antennae in a Rectangular Resonator
}

\author{
Sergey Tkachenko, Jürgen Nitsch, and Moawia Al-Hamid \\ Otto-von-Guericke-University Magdeburg, IGET, Universitätsplatz 2, D-39106 Magdeburg, Germany \\ Correspondence should be addressed to Sergey Tkachenko, sergey.tkachenko@e-technik.uni-magdeburg.de
}

Received 9 September 2011; Accepted 1 December 2011

Academic Editor: Dragan Poljak

Copyright ( $\odot 2012$ Sergey Tkachenko et al. This is an open access article distributed under the Creative Commons Attribution License, which permits unrestricted use, distribution, and reproduction in any medium, provided the original work is properly cited.

The integral-differential equation for the current of an electrically small antenna, inside a resonator, which is induced by given sources, is approximately solved by the so-called "Method of Small Antenna," both for dipole and loop antennas. The current induced in the antenna is evaluated using the scattering characteristics of small antennas in free space and regularized Green's function of resonator. As example of application of the theory, a transfer function ("external field $\rightarrow$ induced voltage") for the coupling through aperture is calculated.

\section{Introduction}

Investigation of the coupling of high-frequency electromagnetic fields caused by intentional electromagnetic interferences to linear structures becomes an actual topic. Usually the corresponding test experiments and simulation models are applied to devices in free space [1]. However, in reality, electronic equipment is enclosed in different kinds of resonator-like shells: cabinets of computers, airframes, frames of cars, and so forth. These enclosures change the interaction of electromagnetic fields with the scatterers remarkably due to rereflections of electromagnetic fields inside the resonator [2].

It was shown in many experiments that the main mechanism of such interaction in free space is electromagnetic coupling to interconnections of different scales. Often these interconnections are electrically small (printed circuit boards, chips, etc.) but can have own resonances. Currents and voltages induced in such objects in free space can be evaluated by a method, which includes two simple models: a model of a small near-resonance linear antenna to describe the common mode and a model of a small loop to describe the differential mode [3].

In our papers, $[4,5]$, we proposed a method to analyze the coupling to an electrically short dipole (or monopole) antenna in a resonator (Method of Small Antenna, MSA) by consequently using the scattering theory. The MSA is based on the analysis of the integro-differential equation describing the induced current in the neighborhood of the antenna. This approach gives the possibility to analytically express the solution for the scattered current in the small antenna inside the resonator from the free space solution and the regularized cavity Green's function. One can also investigate the input impedance of the small antenna, the current transfer ratio for two small antennas, and so forth.

In the present, work we expand our method for the case of electromagnetic coupling to an electrically small loop in the resonator. Again, using an approximate solution for the induced current in free space and a regularized cavity Green's function, we derive an equation for the induced current in an electrically small loop in a resonator. The solution looks like the one for free space but contains a so-called "resonator impedance," instead the radiation resistance of free space. This "resonator impedance" depends on the parameters of the antenna, the parameters of the resonator, and also the coordinates of the loop.

The developed theory was applied to investigate an important practical problem: the calculation of the transfer function (external electromagnetic field $\rightarrow$ scattered current) for electrically small wiring objects (printed circuit, chip, etc.) inside a rectangular resonator. It is assumed that electromagnetic waves penetrate into the cavity through 


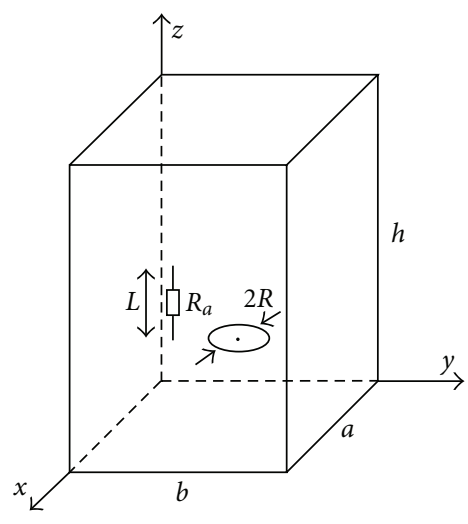

FIGURE 1: Electrical and magnetic dipoles in a resonator.

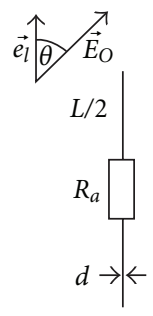

(a)

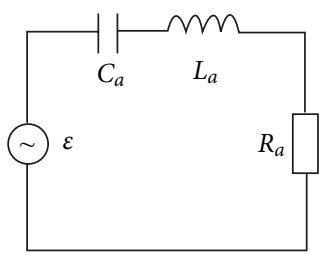

(b)
Figure 2: Model of a small near-resonance electrical dipole antenna.

a small aperture and excite the scattering objects. We show that the analytical evaluations are in good agreement with results of numerical simulation.

The paper is organized as follows: in the Section 2, we shortly repeat main results of MSA for the small electrical dipole antenna in a resonator. In the Section 3, we present new results of MSA for the small loop inside the resonator. In the Section 4, we calculate transfer functions for both type of scatterers and compare our results with numerical calculations. In the short conclusion, we outline directions of future investigations.

\section{Small Electrical Dipole Antenna in a Rectangular Resonator}

We consider a thin-wire (electrical) dipole antenna inside a rectangular cavity with well-conducting walls, see Figure 1, which is excited by an electric field $\vec{E}_{0}(\vec{r})$.

For simplicity, we assume that the antenna is parallel to the $z$-axis. The current induced in the antenna satisfies the electric field integral equation (EFIE), which contains the tensor (dyad) Green's function of the cavity $[4,5]$. The same EFIE equation for the current induced in the excited antenna, can be written in free space. Of course, in this case it contains the Green's function for free space. For an electrically small antenna the EFIE equation in free space can be solved by the approximation of a small near-resonance (electrical) dipole antenna [3], see Figure 2

$$
\begin{gathered}
J_{E}(j \omega, l) \approx E_{z} K_{E}(j \omega) f(l)=\varepsilon_{E, \text { eff }} Y_{E}(j \omega) f(l), \\
Y_{E}(j \omega)=\frac{1}{1 / j \omega C_{a}+j \omega L_{a}+R_{a}} .
\end{gathered}
$$

For a thin symmetric dipole antenna [3],

$$
\begin{gathered}
\omega_{a} \approx \frac{\pi c}{L}, \\
C_{a}=\frac{\pi \varepsilon_{0} L / 2}{\ln (2 L / d)-2}, \\
L_{a}=\frac{1}{C_{a} \omega_{a}^{2}} .
\end{gathered}
$$

Here the quantities $L, d$ are the antenna length and diameter, respectively, $\omega_{a}$ is the first resonance frequency of the antenna, $C_{a}$ and $L_{a}$ are the antenna capacitance and inductance, respectively, and $R_{a}$ is the antennaload.

The effective electromotive force $\varepsilon_{E \text {,eff }}$ and the current distribution function $f(l)$ for a passive antenna with a distributed uniform excitation $E_{z}\left(R_{a} \ll \sqrt{L_{a} / C_{a}}\right)$, see Figure 2(a) are given by (3) and (4), respectively [3-5],

$$
\varepsilon_{E, \text { eff }}=E_{z} \frac{L}{2},
$$

$$
f(l) \approx 1-\frac{4 l^{2}}{L^{2}} .
$$

By the analysis of the EFIE for the small antenna in a resonator, it can be shown that the induced current is described by a simple equation like (1), which, however, contains together with the load resistance $R_{a}$ also the "impedance of the resonator" $Z_{\mathrm{RES}}$. This value is calculated using the resonator Green's function, regularized for coinciding arguments, $\widetilde{\bar{G}}_{\mathrm{RES}}^{E}$, and it depends on both the antenna and resonator parameters. For the passive dipole (distributed excitation), one obtains (detailed derivation and explanation of expression (5) the reader can find in $[3,4]$ )

$$
\begin{aligned}
\tilde{J}_{E}(l) & \approx E_{z} \tilde{K}_{E}(j \omega) f(l)=0.5 E_{z} L \tilde{Y}_{E}(j \omega) f \cdot(l), \\
\tilde{Y}_{E} & =\frac{1}{1 / j \omega C_{a}+j \omega L_{a}+R_{\mathrm{load}}+Z_{\mathrm{RES}}^{E}(j \omega)}, \\
Z_{\mathrm{RES}}^{E}(j \omega) & =-\left(\overline{\bar{G}}_{\mathrm{RES}}^{E}\right)_{Z Z} F^{\text {d.e. }} \frac{L}{2}, \\
F^{\text {d.e. }} & :=\int_{L / 2}^{L / 2} f^{\text {d.e. }}(l) d l=\frac{2}{3 L},
\end{aligned}
$$




$$
\begin{aligned}
\left(\overline{\bar{G}}_{\mathrm{RES}}^{E}\right)_{Z Z} \approx \frac{\eta_{0}}{J K}\{ & \frac{4}{a b} \sum_{n_{x}, n_{y}=1}^{\gamma \leq \gamma_{\max }}\left(\left(k_{x}^{v}\right)^{2}+\left(k_{y}^{v}\right)^{2}\right) \\
& \times \sin ^{2}\left(k_{y}^{v} x\right) \sin ^{2}\left(k_{y}^{v} y\right) \\
& \times \frac{\operatorname{ch}\left(\gamma_{v} / h-z\right) \operatorname{ch}(\gamma h)}{\gamma \operatorname{sh}(\gamma h)} \\
& \left.+\frac{1}{4 \pi}\left(-\gamma_{\max } k^{2}-\frac{\gamma_{\max }^{3}}{3}\right)\right\}, \\
k_{x}^{v}= & \frac{\pi n_{x}}{a}, \\
k_{y}^{v}= & \frac{\pi n_{y}}{b}, \\
\gamma_{v}= & \sqrt{k_{x}^{2}+k_{y}^{2}-k^{2}}, \\
k= & \frac{\omega}{c} \cdot\left(\frac{1-j}{2 Q}\right), \\
\left|\gamma_{\max }\right| & 2.5 k .
\end{aligned}
$$

An analogous simple equation for the induced current can be derived for the lumped excitation [5].

The input impedance of a small dipole antenna in a rectangular resonator was also calculated in [6] to investigate statistical properties. In this work, a different split of the resonator's Green's function was used, taking advantage of the Coulomb gauge for the vector and scalar potential. This was combined with the assumption of a fixed linear free-space current distribution in the antenna for lumped excitation.

\section{Small Magnetic Dipole (Loop) Antenna in Rectangular Resonator}

Consider an electrically small loop inside a rectangular cavity (see Figure 1) with well-conducting walls. For simplicity we assume that the plane of the loop is normal to the $z$ axis. The loop interacts with the magnetic field which is excited in the resonator by one or another way (an additional internal radiating antenna, penetration of a field into the cavity through apertures, etc.). This field induces a current $J$ in the loop, which, in turn, serves as a source of the scattered electromagnetic field. This small loop is equivalent to a magnetic dipole with dipole moment $M=J \cdot S$, where $S$ is the equivalent area of the loop.

For a more simple description of the electromagnetic field of a small loop using the mathematical apparatus of Green's functions, it is convenient to formally introduce a magnetic charge $J_{M}$ and a magnetic current $\rho_{M}$ (see, for example, [7]). The dipole moment of the small loop is connected with the integral of magnetic current by

$$
\int \overrightarrow{J_{M}}(\vec{r}) d V=j \omega \vec{M} \text {. }
$$

Then the electrical and magnetic field in the resonator can be written as

$$
\begin{gathered}
\vec{E}=-\operatorname{rot} \vec{A}^{M}, \\
\vec{H}=\frac{1}{j \omega \mu_{0}}\left(\operatorname{grad} \operatorname{div} \vec{A}^{M}+k^{2} \vec{A}^{M}\right),
\end{gathered}
$$

where

$$
\vec{A}^{M}=\int \overline{\bar{G}}^{A, M}(\vec{r}, \vec{r}) \vec{J}^{M}(\vec{r}) d^{3} \vec{r}
$$

is the vector potential caused by the magnetic current; $\overline{\bar{G}}{ }^{A, M}\left(\vec{r}, \vec{r}^{\prime}\right)$ is the tensor Green's function for the vector potential. This Green's function has a simple diagonal form in free space

$$
\overline{\bar{G}}_{f \cdot s p .}^{A, M}\left(\vec{r}, \vec{r}^{\prime}\right)=\frac{\mu_{0}}{4 \pi} \frac{\exp \left(-j k\left|\vec{r}, \vec{r}^{\prime}\right|\right)}{\left|\vec{r}, \vec{r}^{\prime}\right|} \overline{\bar{I}} .
$$

In the resonator, this Green's function can be calculated by a different way. Here, as in our early research of the small electrical dipole [4], it is convenient to use the resonator Green's function in double sum representation (expansion into transverse waveguide modes of the cavity [7]). It is a diagonal tensor, whose $z z$-component is (the other components can be obtained by cyclic rearrangement) given by

$$
\begin{aligned}
\left(\overline{\bar{G}}_{\mathrm{RES}}^{A, M}\right)_{z z}= & \frac{\mu_{0}}{a b} \sum_{n_{x}, n_{y}=0}^{\infty} \varepsilon_{0, n_{x}} \varepsilon_{0, n_{y}} \cos \left(k_{x}^{v} x\right) \\
& \times \cos \left(k_{x}^{v} x^{\prime}\right) \cos \left(k_{y}^{v} y\right) \cos \left(k_{y}^{v} y^{\prime}\right) \\
& \times \frac{1}{\gamma_{\nu} \sinh \left(\gamma_{\nu} h\right)} \\
& \times \begin{cases}\sinh \left(\gamma_{\nu} z\right) \sinh \left(\gamma_{\nu}\left(h-z^{\prime}\right)\right), & z \geq z^{\prime} \\
\sinh \left(\gamma_{\nu} z^{\prime}\right) \sinh \left(\gamma_{\nu}(h-z)\right), & z^{\prime} \geq z\end{cases}
\end{aligned}
$$

with

$$
\begin{gathered}
k_{x}^{v}=\frac{\pi n_{x}}{a}, \\
k_{y}^{v}=\frac{\pi n_{y}}{b}, \\
\gamma_{\nu}=\sqrt{\left(k_{x}^{v}\right)^{2}+\left(k_{y}^{v}\right)^{2}-k^{2}} \\
\varepsilon_{0, n}=1 \text { for } n=0,=2 \text { for } n \neq 0, \text { and so on. }
\end{gathered}
$$

The magnetic and electrical field can be obtained in an analogous way after integration with the Green's function for the magnetic and electrical fields, respectively, using (8) or (9) and applying (7a) and (7b).

Let us now investigate properties of the Green's functions in the neighborhood of the small scatterer. To make our 
method clearer, we begin from the case in free space. The $z z$-component of the Green's function for the magnetic field from (8) and (9) is approximated by

$$
\begin{aligned}
& \left.\left(\overline{\bar{G}}_{f, s p .}^{H,{ }^{\prime}}\right)_{z z}\right|_{\substack{x=x_{1} \\
y=y_{1} \\
k\left|z-z^{\prime}\right| \ll 1}} \\
& \approx \frac{1}{4 \pi} \frac{1}{j \omega}\left[\frac{2}{\left|z-z^{\prime}\right|^{3}}+\frac{k_{0}^{2}}{\left|z-z^{\prime}\right|^{2}}-\frac{2}{3} j k_{0}^{3}\right] \\
& =\left(\overline{\bar{G}}_{\text {sing. }}^{H, M}\left(z-z^{\prime}\right)\right)_{z z}+\left({\stackrel{\overline{\bar{G}}}{H, M})_{z z} .} .\right.
\end{aligned}
$$

As one can see, the Green's function is decomposed into two parts. The first, singular part (first and second term in the brackets) represents the near field and contains electrostatic and magnetostatic energy. The second, regular part (third term in the bracket) is constant in the neighborhood of the scatterer and is responsible for the far field (radiation for the case of free space, see below).

In order to extract the divergence of the Green's function inside the resonator in explicit form, we divide the summation domain of (9) into two parts [4] (see Figure 4): one corresponding to the values of wave number $\gamma_{v}$ (less than some value $\left.\gamma_{\max }\right)$ and the other part contains those values, which exceed $\gamma_{\max }$. Then the summation may be approximated by integration (practical numerical calculation has shown that a good choice for $\gamma_{\max }$ is $\sim 2.5 k$ ). As a result, we obtain the following expression for $\left(\overline{\bar{G}}_{\mathrm{RES}}^{A, M}\right)_{z z}$ in the vicinity of the scatterer:

$$
\begin{aligned}
& \left(\overline{\bar{G}}_{\mathrm{RES}}^{H, M}\right)_{z z} \mid \begin{array}{c}
x=x_{1} \\
y=y_{1} \\
k\left|z-z^{\prime}\right| \ll 1
\end{array} \\
& \approx \frac{\mu_{0}}{a b} \sum_{n_{1}, n_{2}=0}^{\gamma_{v} \leq \gamma_{\max }} \cdots+\frac{\mu_{0}}{4 \pi} \frac{\exp \left(-\gamma_{\max }\left|z-z^{\prime}\right|\right)}{\left|z-z^{\prime}\right|} .
\end{aligned}
$$

Now, substituting (12) into (7b), going to the limit $k \mid z-$ $z^{\prime} \mid \rightarrow 0$ and keeping main terms, including constant ones, we obtain the desired decomposition of the resonator Green's function for the magnetic field:

$$
\begin{aligned}
&\left(\overline{\bar{G}}_{\mathrm{RES}}^{H, M}\right)_{z z} \mid \begin{array}{c}
x=x_{1} \\
y=y_{1} \\
k\left|z-z^{\prime}\right| \ll 1
\end{array} \\
& \approx \frac{1}{j \omega a b} \sum_{n_{x}, n_{y}=0}^{\gamma_{\nu} \leq \gamma_{\max }} \varepsilon_{o, n_{x}} \varepsilon_{o, n_{y}}\left(\left(k_{x}^{v}\right)^{2}+\left(k_{y}^{v}\right)^{2}\right) \\
& \times \cos ^{2}\left(k_{x}^{v} x\right) \cos ^{2}\left(k_{y}^{v} y\right) \\
& \times \frac{\sinh \left(\gamma_{\nu} z\right) \sinh \left(\gamma_{\nu}(h-z)\right)}{\gamma_{\nu} \sinh \left(\gamma_{\nu} h\right)}+\frac{1}{4 \pi} \frac{1}{j \omega} \\
& \times\left[\frac{2}{\left|z-z^{\prime}\right|^{3}}+\frac{k_{0}^{2}}{\left|z-z^{\prime}\right|^{2}}-\frac{\gamma_{\max }^{3}}{3}-k^{2} \gamma_{\max }\right] .
\end{aligned}
$$

Again, as for the case of free space, we can extract from (13) the singular near field part (the first and the second terms in

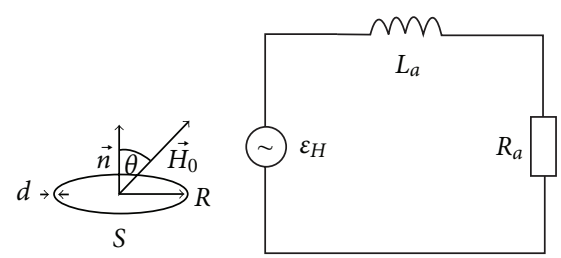

(a)

(b)

Figure 3: Model of a small loop.

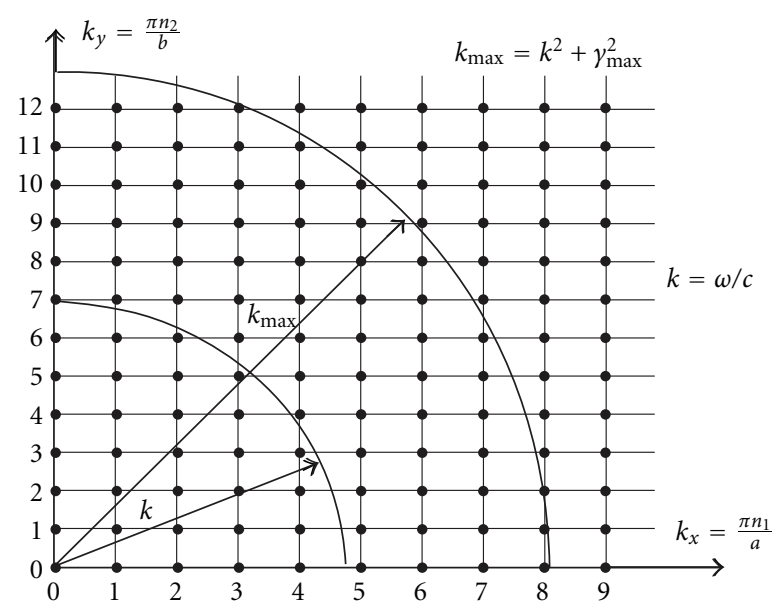

FIGURE 4: Division of the summation domain.

the square brackets), which look like that one in free space and the regular far field part (the sum and the third and fourth terms in the square brackets, which take into account rereflection of the signal from the cavity walls and contain all information about system resonances).

The solution of the interaction problem with the singular part of the Green's function can be obtained by the model of a small loop [3] (see Figures 3(a) and 3(b))

$$
\begin{gathered}
J_{H}(j \omega) \approx \varepsilon_{H, \mathrm{eff}} Y_{H}(j \omega)=K_{H}(j \omega) H_{z}, \\
\varepsilon_{H, \mathrm{eff}}=-j \omega H_{z} \mu_{0} S \\
Y_{H}(j \omega)=\frac{1}{j \omega L_{a}+R_{a}}
\end{gathered}
$$

Here the quantities $S, L_{a}$ are the antenna area and inductance, and $R_{a}$ is the antenna load. For a thin circular loop antenna with radius $R$ and diameter of the wire $d$ [3],

$$
L_{a}=\mu_{0} R\left(\ln \left(\frac{16 R}{d}\right)-2\right)
$$

Let now the loop be under the action of an external magnetic field $H_{z}$. If we take into account only the singular part of the Green's function (11) or (13), the induced current is defined by (14a), (14b) and (14c). But, if we would like to take into 
account the regular part of the Green's function, we have to add to the magnetic field in (14a) an additional part $\tilde{H}_{z}$

$$
\begin{aligned}
\tilde{H}_{z} & =\int\left(\stackrel{\overline{\bar{G}}}{H, M}^{H z} J_{z}^{m}(\vec{r}) d V^{\prime}=\left(\stackrel{\overline{\bar{G}}}{H, M}_{z z} \int J_{z}^{m}(\vec{r}) d V^{\prime}\right.\right. \\
& =\left(\stackrel{\overline{\bar{G}}}{ }^{H, M}\right)_{z z} j \omega M_{z}=\left(\stackrel{\overline{\bar{G}}}{ }^{H, M}\right)_{z z} j \omega S J
\end{aligned}
$$

Here we take into account (11), (6) and the connection of the magnetic moment with the electrical current. Then, from (14a), we have

$$
{\widetilde{J_{H}}}=\left(H_{z}+(\stackrel{\overline{\bar{G}}}{H, M})_{z z} j \omega S{\tilde{J_{H}}}\right) \cdot K_{H}(j \omega) .
$$

This is a linear algebraic equation for the induced current ${\widetilde{J_{H}}}$ whose solution yields

$$
\begin{aligned}
\tilde{J}_{H} & =\frac{K_{H}(j \omega)}{1-\left(\widetilde{G}^{H, M}\right)_{z z} j \omega S K_{H}(j \omega)} H_{z} \\
& =\frac{-j \omega \mu_{0} S}{j \omega L_{a}+R_{a}-\mu_{0} \omega^{2} S^{2}\left(\widetilde{G}^{H, M}\right)_{z z}} H_{z} \\
& =\widetilde{K}_{H}(j \omega) H_{z} .
\end{aligned}
$$

By this way, taking into account the regular part of the Green's function yields the additional impedance $\tilde{Z}_{a}(j \omega)$ to the antenna resistance $R_{a}$ in (14b)

$$
\tilde{Z}_{a}(j \omega)=-\mu_{0} \omega^{2} S^{2}\left(\widetilde{G}^{H, M}\right)_{z z} .
$$

For free space, with $\left(\widetilde{G}^{H, M}\right)_{z z}$ from (11), (19) yields a well-known equation for the radiation resistance of the electrically small loop [3]:

$$
\widetilde{Z}_{a}^{\text {free space }}(j \omega)=\frac{\eta_{0} k^{4} S^{2}}{6 \pi} .
$$

This agreement shows the correctness of our method for a small loop.

For the loop inside the resonator, (19) gives an impedance value for the antenna ("impedance of loop-resonator system") $\widetilde{Z}_{a}^{\mathrm{RES}}$, similar to the case of a small near-resonance dipole in Section 2. This value depends on both, the loop antenna and resonator parameters, and describes all resonances of the following system:

$$
\begin{aligned}
\widetilde{Z}_{a}^{\mathrm{RES}}(j \omega) \approx j \eta_{0} k S^{2}\left\{\frac{1}{a b} \sum_{n_{x}, n_{y}=0}^{\gamma_{v} \leq \gamma_{\max }} \varepsilon_{o, n_{x}} \varepsilon_{o, n_{y}}\left(\left(k_{x}^{v}\right)^{2}+\left(k_{y}^{v}\right)^{2}\right)\right. \\
\quad \times \cos ^{2}\left(k_{x}^{v} x\right) \cos ^{2}\left(k_{y}^{v} y\right) \\
\times \frac{\sinh \left(\gamma_{\nu} z\right) \sinh \left(\gamma_{v}(h-z)\right)}{\gamma_{\nu} \sinh \left(\gamma_{\nu} h\right)} \\
\left.-\frac{1}{4 \pi}\left[-\frac{\gamma_{\max }^{3}}{3}-k^{2} \gamma_{\max }\right]\right\} .
\end{aligned}
$$

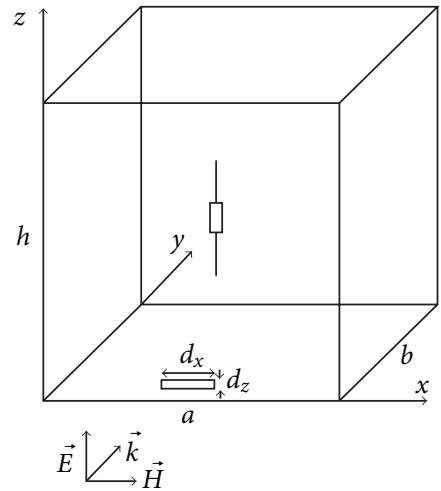

FIGURE 5: Excitation of the internal dipole antenna through small aperture.

Note, that the (18)-(21) in the present section describe, namely, the differential current mode in the loop. This mode prevails for small frequencies, much smaller than the frequency of the first resonance (for the circular loop $\omega_{1} \approx$ $c / R)$. For the higher frequencies to evaluate the total current, one has to take into account the common current mode, which can be obtained using the approach of small electrical dipole antenna described in previous section. Combination of these two modes (depending from the orientation of the loop) gives a total current, which can be compared, for example, with numerical calculations. Such comparison will be a subject of nearest investigations.

\section{Application of the Developed Theory: Transfer Function "External Field-Induced Current"}

Now it is assumed that an exterior field penetrates through a rectangular aperture into the cavity and excites a small dipole (see Figure 5). The dimensions of the resonator are $a=h=$ $0.79 \mathrm{~m}, b=0.534 \mathrm{~m}$. The center of the $z$-directed unloaded dipole antenna with length $L=0.25 \mathrm{~m}$ and radius $r=1 \mathrm{~mm}$ is placed in the point with coordinates $x=0.395 \mathrm{~m}, y=$ $0.3 \mathrm{~m}$, and $z=0.295 \mathrm{~m}$. The cavity is excited through the rectangular slit by the normally incident plane wave with amplitude $E_{0}=100 \mathrm{~V} / \mathrm{m}$. The position of the center of the slit is $x_{1}=0.395 \mathrm{~m}, z_{1}=5 \mathrm{~mm}$; the dimensions of the slit are $d_{x}=100 \mathrm{~mm}, d_{z}=5 \mathrm{~mm}$.

The aperture is modeled by two equivalent electric and magnetic dipoles placed in the center of the aperture [7]. For the considered case, only the $x x$ component of the magnetic dipole moment $M_{x}=-\beta_{x x} H_{x}^{\text {sc }}$ of the aperture is important, which can be calculated using the magnetic polarisability $\beta_{x x}=\pi / 24 d_{x}^{3}\left(\ln \left(4 d_{x} / d_{z}\right)-1\right)^{-1}$. Here $H_{x}^{\text {sc }}$ is a short-circuit magnetic field because the plane of the aperture is assumed to be perfectly conducting when solving the external problem of diffraction. Now using the numerical data for the $H_{x}^{\text {sc }}$, we can calculate the magnetic moment of the aperture. Then we calculate the internal electrical field using $(7 \mathrm{a})$ and $(7 \mathrm{c})$, and (9) and, after that, calculate the current amplitude in the dipole antenna using the results of Section 2. Comparison 


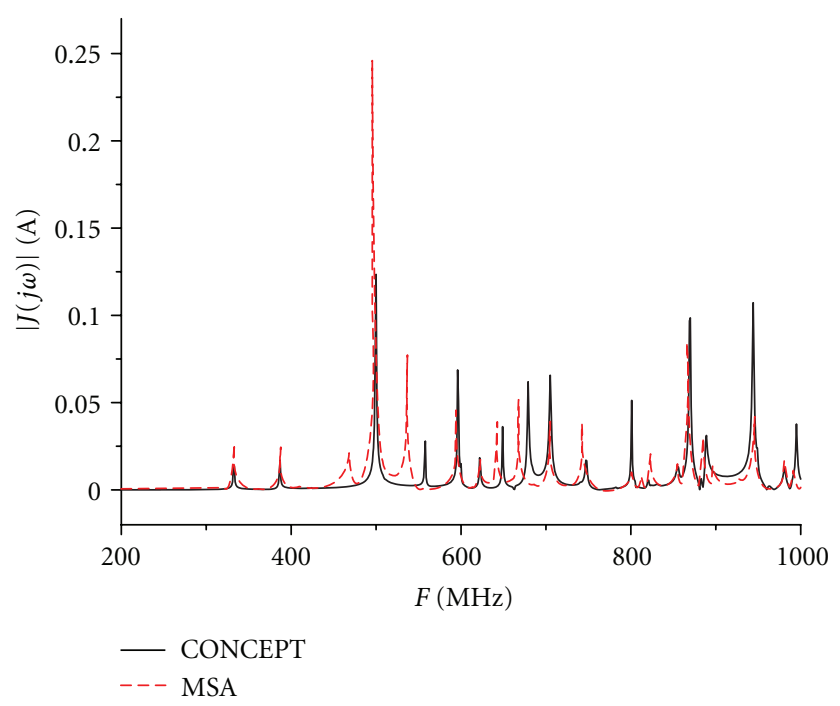

Figure 6: Comparison of results of MSA with CONCEPT.

of the MSA results with those of the well-known numerical code "CONCEPT" is presented in Figure 6. One can see a satisfactory agreement of the numerical and analytical results, especially in the frequency region up to the first resonance of the small antenna. The observed differences (especially the additional peaks near the main resonance) are due to the fact that in this example, taken from a practical HPM coupling problem, the conditions of applicability of the method of small antennas are not well satisfied: the size of the antenna is comparable with the wavelength and the cavity dimensions. Moreover, there may be additional resonances in the penetration of radiation through the slit.

Note, that the calculation of MSA requires about one minute of calculation by the PC notebook (Processor T7250 $2 \mathrm{GHz}$ ); at the same time direct CONCEPT calculation requires approximately one week of calculations by the PC cluster (6 PCs).

\section{Conclusion}

Interactions of high-frequency electromagnetic fields with small scatterers inside rectangular (resonators taking into account both common and differential modes) were investigated using the Method of Small Antenna. The results are important for the study of natural and intentional electromagnetic interferences with printed circuits, chips, and so forth inside racks and housings. Comparison of the results with numerical ones has shown a good agreement. In future, we intend to investigate effective damping of the interferences inside cavities using the generalization of the developed model for the multiscatterer case.

\section{Acknowledgment}

This work was supported by the German Research Foundation under the Contract NI 633/5-1.

\section{References}

[1] M. Camp and H. Garbe, "Susceptibility of personal computer systems to fast transient electromagnetic pulses," IEEE Transactions on Electromagnetic Compatibility, vol. 48, no. 4, pp. 829833, 2006.

[2] M. Magg and J. B. Nitsch, "HPM coupling to genetic targets: CW-vs pulse coupling," in Proceedings of the 11th International Zurich Symposium and Technical Exhibition on Electromagnetic Compatibility, pp. 361-367, March 1995.

[3] V. I. Kravchenko, E. A. Bolotov, and N. I. Letunova, Communication Facilities and Powerful Electromagnetic Interferences, Radio I Svjaz, Moscow, Russia, 1987.

[4] S. V. Tkachenko, G. V. Vodopianov, and L. M. Martinov, "Electromagnetic field coupling to an electrically small antenna in a rectangular cavity," in Proceedings of the 13th International Zurich Symposium and Technical Exhibition on Electromagnetic Compatibility, pp. 379-384, February 1999.

[5] S. Tkachenko, F. Gronwald, H. G. Krauthäuser, and J. Nitsch, "High frequency electromagnetic field coupling to small antennas in rectangular resonator," in Proceedings of the International Conference on Electromagnetics in Advanced Applications (ICEAA '09), pp. 188-191, September 2009.

[6] L. K. Warne, K. S. H. Lee, H. G. Hudson, W. A. Johnson, R. E. Jorgenson, and S. L. Stronach, "Statistical properties of linear antenna impedance in an electrically large cavity," IEEE Transactions on Antennas and Propagation, vol. 51, no. 5, pp. 978-991, 2003.

[7] G. T. Markov and A. F. Chaplin, Excitation of Electromagnetic Waves, Radio I Svjaz, Moscow, Russia, 1983. 

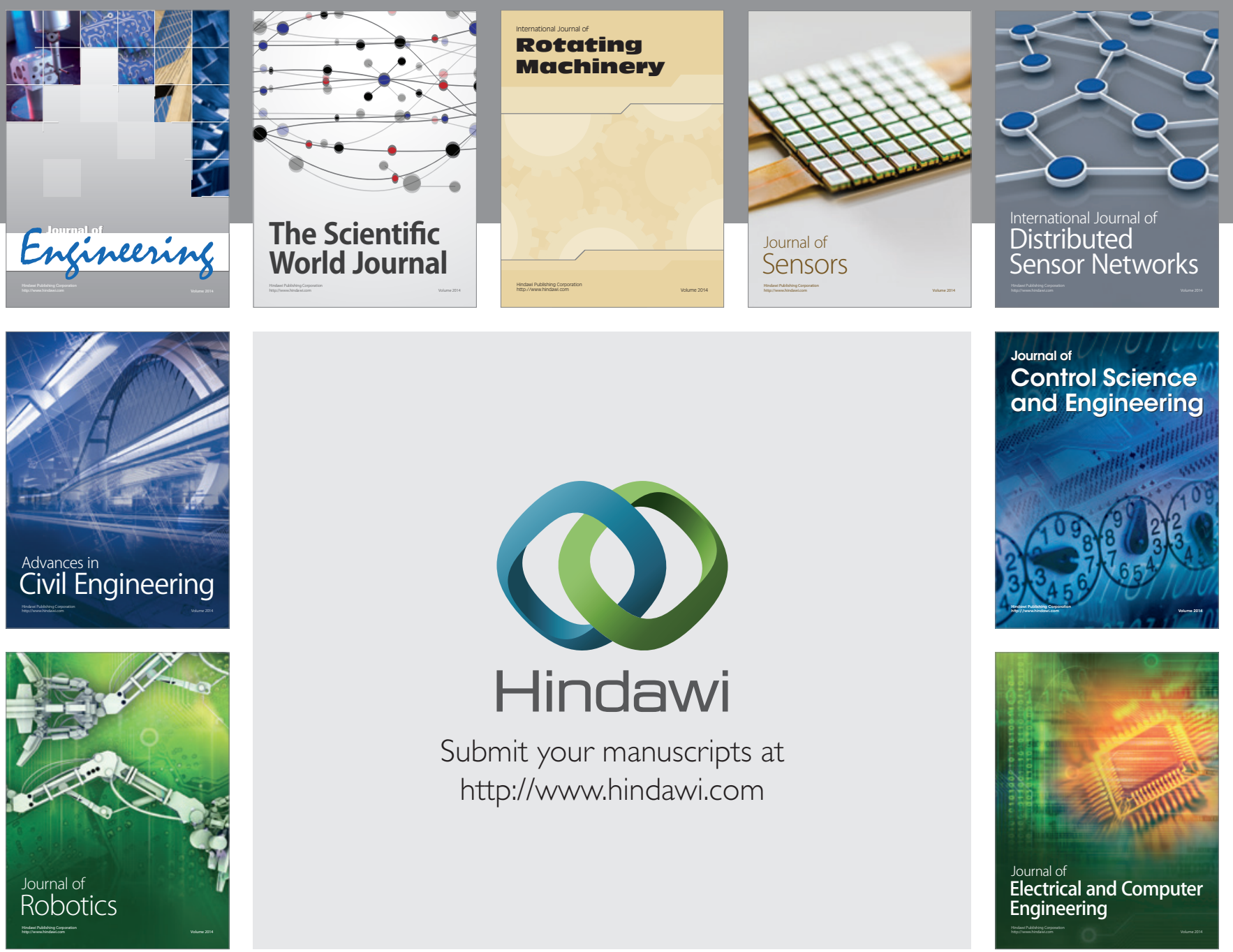

Submit your manuscripts at

http://www.hindawi.com
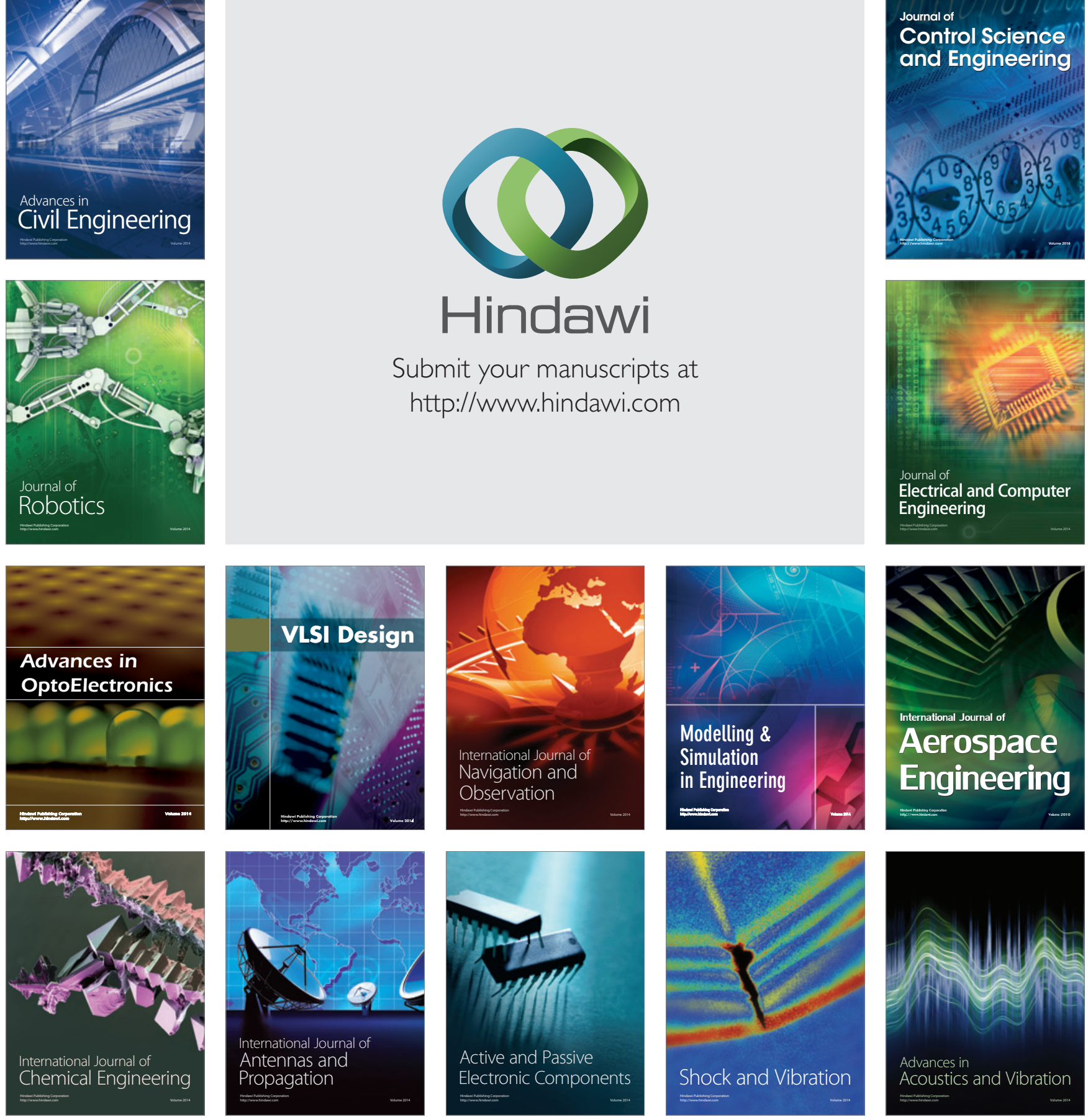